Mathematical Modelling and Analysis

Volume 22 Number 1, January 2017, 1-20

https://doi.org/10.3846/13926292.2017.1258014

(c) Vilnius Gediminas Technical University, 2017
Publisher: Taylor\&Francis and VGTU

http://www.tandfonline.com/TMMA

ISSN: $1392-6292$

eISSN: $1648-3510$

\title{
Space-Time Spectral Collocation Algorithm for the Variable-Order Galilei Invariant Advection Diffusion Equations with a Nonlinear Source Term
}

\author{
Mohamed A. Abd-Elkawy ${ }^{a, b}$ and Rubayyi T. Alqahtani ${ }^{a}$ \\ ${ }^{a}$ Department of Mathematics and Statistics, College of Science, Al-Imam \\ Mohammad Ibn Saud Islamic University (IMSIU) \\ Riyadh, Saudi Arabia \\ ${ }^{b}$ Department of Mathematics, Faculty of Science, Beni-Suef University \\ Beni-Suef, Egypt \\ E-mail(corresp.): melkawy@yahoo.com \\ E-mail: rr-gahtani@hotmail.com
}

Received March 23, 2016; revised October 26, 2016; published online January 5, 2017

\begin{abstract}
This paper presents a space-time spectral collocation technique for solving the variable-order Galilei invariant advection diffusion equation with a nonlinear source term (VO-NGIADE). We develop a collocation scheme to approximate VONGIADE by means of the shifted Jacobi-Gauss-Lobatto collocation (SJ-GL-C) and shifted Jacobi-Gauss-Radau collocation (SJ-GR-C) methods. We successfully extend the proposed technique to solve the two-dimensional space VO-NGIADE. The discussed numerical tests illustrate the capability and high accuracy of the proposed methodologies.
\end{abstract}

Keywords: variable-order Galilei invariant advection diffusion equation, fractional calculus, collocation method, Gauss-Radau quadrature, Gauss-Lobatto quadrature.

AMS Subject Classification: 42C05; 65D99; 35R11; 65N35.

\section{Introduction}

In recent years, spectral methods (see [4,10,21] ) are often efficient and highly accurate schemes when compared with the local methods. The speed of convergence is one of the great advantages of spectral methods. Besides, spectral methods have exponential rates of convergence; they also have high level of accuracy. The main idea of all versions of spectral methods is to express the approximate solution of the problem as a finite sum of certain basis functions (orthogonal polynomials or combination of them) and then choose the coefficients in order to minimize the difference between the exact and approximate 
solutions as well as possible. The spectral collocation method is a specific type of spectral methods, that is more applicable and widely used to solve almost types of differential equations $[2,3]$.

Fractional calculus $[1,19,28]$ is a branch of calculus theory, which makes partial differential equations (PDEs) more convenient to describe many phenomena in several fields such as fluid mechanics, chemistry [12,15], biology [19], viscoelasticity [20], engineering, finance and physics [14] fields. The concept of variable-order fractional allows the power of the fractional operator to be a function of the independent variable. The early studies of variable-order fractional was firstly introduced by Samko and Ross [22] and Lorenzo and Hartley $[17,18]$. Several phenomena can be more accurately described using variable-order fractional operators. Mechanical [9], diffusion [5, 26, 34], FIR filters [29] multifractional Gaussian noises [25] and physical [13] models can be more accurately described by variable order derivatives mathematical models. Few numerical methods have been introduced and discussed to solve the variable-order fractional problems. Stability and convergence of explicit finitedifference method has been studied in [16] for solving the variable-order nonlinear fractional diffusion equation. Using Fourier analysis, Chen [6] obtained the numerical solutions for two-dimensional variable-order modified diffusion equations. Numerical methods based on finite difference techniques [8, 24, 31, 32] have been proposed by Liu, Shen, Zhang et al. for the numerical treatment of variable-order fractional partial differential equations. Zhao et al. [33], introduced two second-order approximation algorithms for the variable-order fractional time derivatives. Also, finite difference method has been applied by Xu and Ertürk [30] to solve the fractional integro-differential equations with variable order. Moreover, finite difference schemes [27] have been introduced to solve variable-order time fractional diffusion equation.

The fractional advection-diffusion equation [11,23] can be considered as a generalized version of the classical advection-diffusion equation. The fractional advection-diffusion equation is used to model many physical phenomena such as amorphous, colloid, the transport dynamics in complex systems, fractals and percolation clusters, biological systems, glassy and porous media, comb structures, dielectrics and semiconductors, polymers, random and disordered media, geophysical and geological processes, the transport of passive tracers carried by fluid flow in a porous medium. Here, we focus on the application of SJ-GL-C and SJ-GR-C schemes to numerically solve the VO-NGIADE in one and two dimensional space. The proposed collocation scheme is investigated for both temporal and spatial discretizations. The SJ-GL-C and SJ-GR-C are proposed, with a suitable modification for treating the boundary and initial conditions, for spatial and temporal discretizations. This treatment, for the conditions, improves the accuracy of the scheme greatly. Therefore, the VO-NGIADE with its conditions is reduced to system of nonlinear algebraic equations which is far easier to be solved. In addition, this algorithm is developed to numerically solve the two-dimensional VO-NGIADE. Thus, we introduce a fully spectral collocation approach to numerically treat the multi-dimensional VO-NGIADE. Moreover, there are no numerical results on the spectral collocation method for solving the VO-NGIADE. Finally, several numerical examples with com- 
parisons lighting the high accuracy and effectiveness of the proposed algorithm are presented.

This paper is organized as follows. Few facts of shifted Jacobi polynomials are listed in Section 2. In Section 3, we introduce a new collocation method for the one-dimensional space VO-NGIADE. In Section 4, the proposed scheme is successfully extended to solve the two-dimensional space VO-NGIADE. Section 5 is customized to solve several problems. Conclusions are given in the last section.

\section{Properties of shifted Jacobi polynomials}

Some few properties of shifted Jacobi polynomials are presented in this section. In the following, few relations related to Jacobi polynomials are listed:

$$
\begin{aligned}
& P_{k+1}^{(\alpha, \beta)}(x)=\left(a_{k}^{(\alpha, \beta)} x-b_{k}^{(\alpha, \beta)}\right) P_{k}^{(\alpha, \beta)}(x)-c_{k}^{(\alpha, \beta)} P_{k-1}^{(\alpha, \beta)}(x), \quad k \geq 1, \\
& P_{0}^{(\alpha, \beta)}(x)=1, \quad P_{1}^{(\alpha, \beta)}(x)=0.5(\alpha+\beta+2) x+0.5(\alpha-\beta), \\
& P_{k}^{(\alpha, \beta)}(-x)=(-1)^{k} P_{k}^{(\alpha, \beta)}(x), \quad P_{k}^{(\alpha, \beta)}(-1)=\frac{(-1)^{k} \Gamma(k+\beta+1)}{k ! \Gamma(\beta+1)},
\end{aligned}
$$

where $\alpha, \beta>-1, x \in[-1,1]$ and

$$
\begin{aligned}
a_{k}^{(\alpha, \beta)} & =\frac{(2 k+\alpha+\beta+1)(2 k+\alpha+\beta+2)}{2(k+1)(k+\alpha+\beta+1)}, \\
b_{k}^{(\alpha, \beta)} & =\frac{\left(\beta^{2}-\alpha^{2}\right)(2 k+\alpha+\beta+1)}{2(k+1)(k+\alpha+\beta+1)(2 k+\alpha+\beta)}, \\
c_{k}^{(\alpha, \beta)} & =\frac{(k+\alpha)(k+\beta)(2 k+\alpha+\beta+2)}{(k+1)(k+\alpha+\beta+1)(2 k+\alpha+\beta)} .
\end{aligned}
$$

Moreover, the $r$ th derivative $\left(r\right.$ is an intger) of $P_{j}^{(\alpha, \beta)}(x)$, may be obtained from

$$
D^{r} P_{j}^{(\alpha, \beta)}(x)=\frac{\Gamma(j+\alpha+\beta+q+1)}{2^{r} \Gamma(j+\alpha+\beta+1)} P_{j-r}^{(\alpha+r, \beta+r)}(x) .
$$

For the shifted Jacobi polynomial $P_{L, k}^{(\alpha, \beta)}(x)=P_{k}^{(\alpha, \beta)}\left(\frac{2 x}{L}-1\right), L>0$, the explicit analytic form is written as

$$
\begin{aligned}
P_{L, k}^{(\alpha, \beta)}(x) & =\sum_{j=0}^{k}(-1)^{k-j} \frac{\Gamma(k+\beta+1) \Gamma(j+k+\alpha+\beta+1)}{\Gamma(j+\beta+1) \Gamma(k+\alpha+\beta+1)(k-j) ! j ! L^{j}} x^{j} \\
& =\sum_{j=0}^{k} \frac{\Gamma(k+\alpha+1) \Gamma(k+j+\alpha+\beta+1)}{j !(k-j) ! \Gamma(j+\alpha+1) \Gamma(k+\alpha+\beta+1) L^{j}}(x-L)^{j} .
\end{aligned}
$$

Thus, we can derive the following properties

$$
\begin{aligned}
& P_{L, k}^{(\alpha, \beta)}(0)=(-1)^{k} \frac{\Gamma(k+\beta+1)}{\Gamma(\beta+1) k !}, \quad P_{L, k}^{(\alpha, \beta)}(L)=\frac{\Gamma(k+\alpha+1)}{\Gamma(\alpha+1) k !}, \\
& D^{r} P_{L, k}^{(\alpha, \beta)}(0)=\frac{(-1)^{k-r} \Gamma(k+\beta+1)(k+\alpha+\beta+1)_{r}}{L^{r} \Gamma(k-r+1) \Gamma(r+\beta+1)}
\end{aligned}
$$




$$
\begin{aligned}
& D^{r} P_{L, k}^{(\alpha, \beta)}(L)=\frac{\Gamma(k+\alpha+1)(k+\alpha+\beta+1)_{r}}{L^{r} \Gamma(k-r+1) \Gamma(r+\alpha+1)} \\
& D^{r} P_{L, k}^{(\alpha, \beta)}(x)=\frac{\Gamma(r+k+\alpha+\beta+1)}{L^{r} \Gamma(k+\alpha+\beta+1)} P_{L, k-r}^{(\alpha+r, \beta+r)}(x) .
\end{aligned}
$$

We used $x_{N, j}^{(\alpha, \beta)}$, and $\varpi_{N, j}^{(\alpha, \beta)}, 0 \leqslant j \leqslant N$, as the nodes and Christoffel numbers of the standard Jacobi-Gauss interpolation on the interval $[-1,1]$.

The corresponding nodes and Christoffel numbers of the shifted JacobiGauss interpolation on the interval $[0, L]$ can be given by

$$
x_{L, N, j}^{(\alpha, \beta)}=0.5 L\left(x_{N, j}^{(\alpha, \beta)}+1\right), \quad \varpi_{L, N, j}^{(\alpha, \beta)}=(0.5 L)^{\alpha+\beta+1} \varpi_{N, j}^{(\alpha, \beta)}, 0 \leqslant j \leqslant N .
$$

\section{One-dimensional space of VO-NGIADE}

In this section, we introduce a numerical algorithm based on the SJ-GR-C and SJ-GL-C methods for solving numerically one-dimensional VO-NGIADE. The collocation points are selected at the SJ-GR and SJ-GL interpolation nodes for temporal and spatial variables, respectively. The core of the proposed method consists of discretizing the one-dimensional VO-NGIADE to create a system of nonlinear algebraic equations of the unknown coefficients. This system can be then easily solved with a standard numerical scheme. In particular, we consider the following VO-NGIADE

$$
\frac{\partial u(x, t)}{\partial t}+\frac{\partial u(x, t)}{\partial x}=D_{t}^{1-\gamma(x, t)}\left(\frac{\partial^{2} u(x, t)}{\partial x^{2}}\right)+H(u(x, t), x, t)
$$

given in $(x, t) \in[0, L] \times[0, T]$ with the initial-boundary conditions

$$
u(x, 0)=g_{1}(x), \quad u(0, t)=g_{2}(t), \quad u(L, t)=g_{3}(t), \quad(x, t) \in[0, L] \times[0, T],
$$

where $H(u(x, t), x, t), g_{1}(x), g_{2}(t)$ and $g_{3}(t)$ are given functions, $0<\gamma(x, t)<$ 1 and $D_{t}^{1-\gamma(x, t)} u(x, t)$ is the temporal fractional derivative of variable order $1-\gamma(x, t)$ in the Riemann-Liouville sense [7, 16,34]:

$$
D_{t}^{1-\gamma(x, t)} u(x, t)=\frac{1}{\Gamma(\gamma(x, t))}\left(\frac{\partial}{\partial \zeta}\left(\int_{0}^{\zeta} \frac{u(x, \tau)}{(\zeta-\tau)^{1-\gamma(x, t)}} d \tau\right)\right)_{\zeta=t} .
$$

We are interested in using the SJ-GL-C and SJ-GR-C methods to transform the previous VO-NGIADE into a system of nonlinear algebraic equations. In order to do this, we approximate the independent space variable $x$ using the SJ-GL-C method at the $x_{L, N, i}^{\left(\alpha_{1}, \beta_{1}\right)}$ nodes, while the independent temporal variable $t$ was approximated by the SJ-GR-C methods. The nodes are the set of points in a specified domain where the dependent variable values are to be approximated. In general, the choice of the location of the nodes is optional. However, taking the roots of the shifted Jacobi orthogonal polynomials, referred to as shifted Jacobi collocation points, gives particularly accurate solutions for the spectral methods. 
Now, we outline the main steps of the mixed SJ-GL-C and SJ-GR-C methods for solving the one-dimensional space VO-NGIADE. We choose the approximate solution to be of the form

$$
u_{N, M}(x, t)=\sum_{i=0}^{N} \sum_{j=0}^{M} a_{i, j} P_{L, i}^{\left(\alpha_{1}, \beta_{1}\right)}(x) P_{T, j}^{\left(\alpha_{2}, \beta_{2}\right)}(t)=\sum_{i=0}^{N} \sum_{j=0}^{M} a_{i, j} f_{0}^{i, j}(x, t),
$$

where $f_{0}^{i, j}(x, t)=P_{L, i}^{\left(\alpha_{1}, \beta_{1}\right)}(x) P_{T, j}^{\left(\alpha_{2}, \beta_{2}\right)}(t)$. Then the spatial partial derivatives $\frac{\partial u(x, t)}{\partial x}$ and $\frac{\partial^{2} u(x, t)}{\partial x^{2}}$ were computed as

$$
\begin{aligned}
& \frac{\partial u(x, t)}{\partial x}=\sum_{i=0}^{N} \sum_{j=0}^{M} a_{i, j} \frac{\partial P_{L, i}^{\left(\alpha_{1}, \beta_{1}\right)}(x)}{\partial x} P_{T, j}^{\left(\alpha_{2}, \beta_{2}\right)}(t)=\sum_{i=0}^{N} \sum_{j=0}^{M} a_{i, j} f_{1}^{i, j}(x, t), \\
& \frac{\partial^{2} u(x, t)}{\partial x^{2}}=\sum_{i=0}^{N} \sum_{j=0}^{M} a_{i, j} \frac{\partial^{2} P_{L, i}^{\left(\alpha_{1}, \beta_{1}\right)}(x)}{\partial x^{2}} P_{T, j}^{\left(\alpha_{2}, \beta_{2}\right)}(t)=\sum_{i=0}^{N} \sum_{j=0}^{M} a_{i, j} f_{2}^{i, j}(x, t),
\end{aligned}
$$

dependence on Eq. (2.2), we obtain

$$
\begin{aligned}
& f_{1}^{i, j}(x, t)=\frac{\alpha_{1}+\beta_{1}+i+1}{L} P_{L, i-1}^{\left(\alpha_{1}+1, \beta_{1}+1\right)}(x) P_{T, j}^{\left(\alpha_{2}, \beta_{2}\right)}(t), \\
& f_{2}^{i, j}(x, t)=\frac{\left(\alpha_{1}+\beta_{1}+i+1\right)\left(\alpha_{1}+\beta_{1}+i+2\right)}{L^{2}} P_{L, i-2}^{\left(\alpha_{1}+2, \beta_{1}+2\right)}(x) P_{T, j}^{\left(\alpha_{2}, \beta_{2}\right)}(t) .
\end{aligned}
$$

Furthermore, the temporal derivative $\frac{\partial u(x, t)}{\partial t}$ is evaluated as

$$
\frac{\partial u(x, t)}{\partial t}=\sum_{i=0}^{N} \sum_{j=0}^{M} a_{i, j} P_{L, i}^{\left(\alpha_{1}, \beta_{1}\right)}(x) \frac{\partial P_{T, j}^{\left(\alpha_{2}, \beta_{2}\right)}(t)}{\partial t}=\sum_{i=0}^{N} \sum_{j=0}^{M} a_{i, j} f_{3}^{i, j}(x, t),
$$

dependence on Eq. (2.2), we obtain

$$
f_{3}^{i, j}(x, t)=\frac{\alpha_{2}+\beta_{2}+i+1}{T} P_{L, i}^{\left(\alpha_{1}, \beta_{1}\right)}(x) P_{T, j-1}^{\left(\alpha_{2}+1, \beta_{2}+1\right)}(t) .
$$

Moreover, the Riemann-Liouville fractional partial derivative of variable order $D_{t}^{1-\gamma(x, t)} \frac{\partial^{2} u(x, t)}{\partial x^{2}}$ is given by

$$
\begin{aligned}
D_{t}^{1-\gamma(x, t)} \frac{\partial^{2} u(x, t)}{\partial x^{2}} & =\sum_{i=0}^{N} \sum_{j=0}^{M} a_{i, j} \frac{\partial^{2} P_{L, i}^{\left(\alpha_{1}, \beta_{1}\right)}(x)}{\partial x^{2}} D_{t}^{1-\gamma(x, t)}\left(P_{T, j}^{\left(\alpha_{2}, \beta_{2}\right)}(t)\right) \\
& =\sum_{i=0}^{N} \sum_{j=0}^{M} a_{i, j} f_{4}^{i, j}(x, t)
\end{aligned}
$$

dependence on Eq. (2.2), we obtain

$$
f_{4}^{i, j}(x, t)=\frac{\left(\alpha_{1}+\beta_{1}+i+1\right)\left(\alpha_{1}+\beta_{1}+i+2\right)}{L^{2}} P_{L, i-2}^{\left(\alpha_{1}+2, \beta_{1}+2\right)}(x) D_{t}^{1-\gamma(x, t)}\left(P_{T, j}^{\left(\alpha_{2}, \beta_{2}\right)}(t)\right) .
$$


Now, adopting (3.2)-(3.3), enable one to write (3.1) in the form:

$$
\begin{aligned}
& \sum_{i=0}^{N} \sum_{j=0}^{M} a_{i, j} f_{3}^{i, j}(x, t)=\sum_{i=0}^{N} \sum_{j=0}^{M} a_{i, j} f_{4}^{i, j}(x, t)-\sum_{i=0}^{N} \sum_{j=0}^{M} a_{i, j} f_{1}^{i, j}(x, t) \\
&+H\left(\sum_{i=0}^{N} \sum_{j=0}^{M} a_{i, j} f_{0}^{i, j}(x, t), x, t\right), \quad(x, t) \in[0, L] \times[0, T]
\end{aligned}
$$

The initial condition immediately gives

$$
u(x, 0)=\sum_{i=0}^{N} \sum_{j=0}^{M} a_{i, j} f_{0}^{i, j}(x, 0)=g_{1}(x),
$$

while the numerical treatments of the boundary conditions are

$$
u(0, t)=\sum_{i=0}^{N} \sum_{j=0}^{M} a_{i, j} f_{0}^{i, j}(0, t)=g_{2}(t), u(L, t)=\sum_{i=0}^{N} \sum_{j=0}^{M} a_{i, j} f_{0}^{i, j}(L, t)=g_{3}(t) .
$$

In the proposed mixed SJ-GL-C and SJ-GR-C methods, the residual of (3.4) is set to zero at $M(N-1)$ of SJ-GL and SJ-GR points. Consequently, we find

$$
\sum_{i=0}^{N} \sum_{j=0}^{M} F_{r, s}^{i, j} a_{i, j}=H\left(\sum_{i=0}^{N} \sum_{j=0}^{M} a_{i, j} f_{0}^{i, j}\left(x_{L, N, r}^{\left(\alpha_{1}, \beta_{1}\right)}, t_{T, M, s}^{\left(\alpha_{2}, \beta_{2}\right)}\right), x_{L, N, r}^{\left(\alpha_{1}, \beta_{1}\right)}, t_{T, M, s}^{\left(\alpha_{2}, \beta_{2}\right)}\right),
$$

for $r=1, \ldots, N-1, s=1, \ldots, M$, where,

$$
F_{r, s}^{i, j}=f_{3}^{i, j}\left(x_{L, N, r}^{\left(\alpha_{1}, \beta_{1}\right)}, t_{T, M, s}^{\left(\alpha_{2}, \beta_{2}\right)}\right)+f_{2}^{i, j}\left(x_{L, N, r}^{\left(\alpha_{1}, \beta_{1}\right)}, t_{T, M, s}^{\left(\alpha_{2}, \beta_{2}\right)}\right)-f_{4}^{i, j}\left(x_{L, N, r}^{\left(\alpha_{1}, \beta_{1}\right)}, t_{T, M, s}^{\left(\alpha_{2}, \beta_{2}\right)}\right) .
$$

Dependence on Eqs. (3.5) and (3.6), we obtain

$$
\begin{aligned}
& \sum_{i=0}^{N} \sum_{j=0}^{M} a_{i, j} f_{0}^{i, j}\left(x_{L, N, r}^{\left(\alpha_{1}, \beta_{1}\right)}, 0\right)=g_{1}\left(x_{L, N, r}^{\left(\alpha_{1}, \beta_{1}\right)}\right), \quad r=1, \ldots, N-1, \\
& \sum_{i=0}^{N} \sum_{j=0}^{M} a_{i, j} f_{0}^{i, j}\left(L, t_{T, M, s}^{\left(\alpha_{2}, \beta_{2}\right)}\right)=g_{2}\left(t_{T, M, s}^{\left(\alpha_{2}, \beta_{2}\right)}\right), \quad s=0, \ldots, M \\
& \sum_{i=0}^{N} \sum_{j=0}^{M} a_{i, j} f_{0}^{i, j}\left(L, t_{T, M, s}^{\left(\alpha_{2}, \beta_{2}\right)}\right)=g_{3}\left(t_{T, M, s}^{\left(\alpha_{2}, \beta_{2}\right)}\right), \quad s=0, \ldots, M .
\end{aligned}
$$

Combining Eqs. (3.7), (3.8), (3.9) and (3.10), we obtain for $r=1, \ldots, N-1$, $s=1, \ldots, M$

$$
\begin{aligned}
& \sum_{i=0}^{N} \sum_{j=0}^{M} F_{r, s}^{i, j} a_{i, j}=H\left(\sum_{i=0}^{N} \sum_{j=0}^{M} a_{i, j} f_{0}^{i, j}\left(x_{L, N, r}^{\left(\alpha_{1}, \beta_{1}\right)}, t_{T, M, s}^{\left(\alpha_{2}, \beta_{2}\right)}\right), x_{L, N, r}^{\left(\alpha_{1}, \beta_{1}\right)}, t_{T, M, s}^{\left(\alpha_{2}, \beta_{2}\right)}\right), \\
& \sum_{i=0}^{N} \sum_{j=0}^{M} a_{i, j} f_{0}^{i, j}\left(x_{L, N, r}^{\left(\alpha_{1}, \beta_{1}\right)}, 0\right)=g_{1}\left(x_{L, N, r}^{\left(\alpha_{1}, \beta_{1}\right)}\right), \quad r=1, \cdots, N-1,
\end{aligned}
$$




$$
\begin{aligned}
& \sum_{i=0}^{N} \sum_{j=0}^{M} a_{i, j} f_{0}^{i, j}\left(L, t_{T, M, s}^{\left(\alpha_{2}, \beta_{2}\right)}\right)=g_{2}\left(t_{T, M, s}^{\left(\alpha_{2}, \beta_{2}\right)}\right), \quad s=0, \cdots, M, \\
& \sum_{i=0}^{N} \sum_{j=0}^{M} a_{i, j} f_{0}^{i, j}\left(L, t_{T, M, s}^{\left(\alpha_{2}, \beta_{2}\right)}\right)=g_{3}\left(t_{T, M, s}^{\left(\alpha_{2}, \beta_{2}\right)}\right), \quad s=0, \cdots, M,
\end{aligned}
$$

the previous system of nonlinear algebraic equations can be easily solved. After the coefficients $a_{i, j}$ are determined, it is straightforward to compute the approximate solution $u_{N, M}(x, t)$ at any value of $(x, t)$ in the given domain from the following equation

$$
u_{N, M}(x, t)=\sum_{i=0}^{N} \sum_{j=0}^{M} a_{i, j} P_{L, i}^{\left(\alpha_{1}, \beta_{1}\right)}(x) P_{T, j}^{\left(\alpha_{2}, \beta_{2}\right)}(t) .
$$

\section{Two-dimensional space of VO-NGIADE}

In the present section, we extend the previous algorithm to numerically solve the two-dimensional space VO-NGIADE in the following form

$$
\begin{gathered}
\frac{\partial u(x, y, t)}{\partial t}=D_{t}^{1-\gamma(x, y, t)}\left(\frac{\partial^{2} u(x, y, t)}{\partial x^{2}}+\frac{\partial^{2} u(x, y, t)}{\partial y^{2}}\right)-\frac{\partial u(x, y, t)}{\partial x} \\
+H(u(x, y, t), x, y, t), \quad(x, y, t) \in\left[0, L_{1}\right] \times\left[0, L_{2}\right] \times[0, T]
\end{gathered}
$$

subject to the initial-boundary conditions

$$
\begin{aligned}
& u(x, y, 0)=g_{0}(x, y), \quad(x, y) \in\left[0, L_{1}\right] \times\left[0, L_{2}\right], \\
& u(0, y, t)=g_{1}(y, t), \quad u\left(L_{1}, y, t\right)=g_{2}(y, t), \quad(y, t) \in\left[0, L_{2}\right] \times[0, T], \\
& u(x, 0, t)=g_{3}(x, t), \quad u\left(x, L_{2}, t\right)=g_{4}(x, t), \quad(x, t) \in\left[0, L_{1}\right] \times[0, T],
\end{aligned}
$$

where $H(u(x, y, t), x, y, t), g_{0}(x, y), g_{1}(y, t), g_{2}(y, t), g_{3}(x, t)$ and $g_{4}(x, t)$ are given real valued functions and $u(x, y, t)$ is an unknown function. Therefore, the SJ-GL-C and SJ-GR-C methods will be applied to transform the previous two-dimensional VO-NGIADE into system of nonlinear algebraic equations. The SJ-GL-C and SJ-GR-C have been used for the space $(x, y)$ and time $t$ approximations, respectively.

Now, we outline the main steps of the collocation method for solving the two-dimensional VO-NGIADE. Let

$$
\begin{aligned}
u_{N, M, K}(x, y, t) & =\sum_{i=0}^{N} \sum_{j=0}^{M} \sum_{k=0}^{K} a_{i, j, k} P_{L_{1}, i}^{\left(\alpha_{1}, \beta_{1}\right)}(x) P_{L_{2}, j}^{\left(\alpha_{2}, \beta_{2}\right)}(y) P_{T, k}^{\left(\alpha_{3}, \beta_{3}\right)}(t) \\
& =\sum_{i=0}^{N} \sum_{j=0}^{M} \sum_{j=0}^{K} a_{i, j, k} f_{0}^{i, j, k}(x, y, t)
\end{aligned}
$$

where $f_{0}^{i, j, k}(x, y, t)=P_{L_{1}, i}^{\left(\alpha_{1}, \beta_{1}\right)}(x) P_{L_{2}, j}^{\left(\alpha_{2}, \beta_{2}\right)}(y) P_{T, k}^{\left(\alpha_{3}, \beta_{3}\right)}(t)$. 
Then the first spatial and temporal partial derivatives $\frac{\partial u(x, y, t)}{\partial x}, \frac{\partial u(x, y, t)}{\partial y}$ and $\frac{\partial u(x, y, t)}{\partial t}$ can be computed as

$$
\begin{aligned}
\frac{\partial u(x, y, t)}{\partial x} & =\sum_{i=0}^{N} \sum_{j=0}^{M} \sum_{k=0}^{K} a_{i, j, k} \frac{\partial P_{L_{1}, i}^{\left(\alpha_{1}, \beta_{1}\right)}(x)}{\partial x} P_{L_{2}, j}^{\left(\alpha_{2}, \beta_{2}\right)}(y) P_{T, k}^{\left(\alpha_{3}, \beta_{3}\right)}(t) \\
& =\sum_{i=0}^{N} \sum_{j=0}^{M} \sum_{j=0}^{K} a_{i, j, k} f_{1}^{i, j, k}(x, y, t), \\
\frac{\partial u(x, y, t)}{\partial y} & =\sum_{i=0}^{N} \sum_{j=0}^{M} \sum_{k=0}^{K} a_{i, j, k} P_{L_{1}, i}^{\left(\alpha_{1}, \beta_{1}\right)}(x) \frac{\partial P_{L_{2}, j}^{\left(\alpha_{2}, \beta_{2}\right)}(y)}{\partial y} P_{T, k}^{\left(\alpha_{3}, \beta_{3}\right)}(t) \\
& =\sum_{i=0}^{N} \sum_{j=0}^{M} \sum_{j=0}^{K} a_{i, j, k} f_{2}^{i, j, k}(x, y, t), \\
\frac{\partial u(x, y, t)}{\partial t} & =\sum_{i=0}^{N} \sum_{j=0}^{M} \sum_{k=0}^{K} a_{i, j, k} P_{L_{1}, i}^{\left(\alpha_{1}, \beta_{1}\right)}(x) P_{L_{2}, j}^{\left(\alpha_{2}, \beta_{2}\right)}(y) \frac{\partial P_{T, k}^{\left(\alpha_{3}, \beta_{3}\right)}(t)}{\partial t} \\
& =\sum_{i=0}^{N} \sum_{j=0}^{M} \sum_{j=0}^{K} a_{i, j, k} f_{3}^{i, j, k}(x, y, t),
\end{aligned}
$$

where

$$
\begin{aligned}
& f_{1}^{i, j, k}(x, y, t)=\frac{\alpha_{1}+\beta_{1}+i+1}{L_{1}} P_{L_{1}, i-1}^{\left(\alpha_{1}+1, \beta_{1}+1\right)}(x) P_{L_{2}, j}^{\left(\alpha_{2}, \beta_{2}\right)}(y) P_{T, k}^{\left(\alpha_{3}, \beta_{3}\right)}(t), \\
& f_{2}^{i, j, k}(x, y, t)=\frac{\alpha_{2}+\beta_{2}+j+1}{L_{2}} P_{L_{1}, i}^{\left(\alpha_{1}, \beta_{1}\right)}(x) P_{L_{2}, j-1}^{\left(\alpha_{2}+1, \beta_{2}+1\right)}(y) P_{T, k}^{\left(\alpha_{3}, \beta_{3}\right)}(t), \\
& \left.f_{3}^{i, j, k}(x, y, t)=\frac{\alpha_{3}+\beta_{3}+k+1}{T} P_{L_{1}, i}^{\left(\alpha_{1}, \beta_{1}\right)}(x)\right) P_{L_{2}, j}^{\left(\alpha_{2}, \beta_{2}\right)}(y) P_{T, k-1}^{\left(\alpha_{3}+1, \beta_{3}+1\right)}(t) .
\end{aligned}
$$

While, the second spatial partial derivatives $\frac{\partial^{2} u(x, y, t)}{\partial x^{2}}$ and $\frac{\partial^{2} u(x, y, t)}{\partial y^{2}}$ are given by

$$
\begin{aligned}
& \frac{\partial^{2} u(x, y, t)}{\partial x^{2}}=\sum_{i=0}^{N} \sum_{j=0}^{M} \sum_{j=0}^{K} a_{i, j, k} f_{4}^{i, j, k}(x, y, t), \\
& \frac{\partial^{2} u(x, y, t)}{\partial y^{2}}=\sum_{i=0}^{N} \sum_{j=0}^{M} \sum_{j=0}^{K} a_{i, j, k} f_{5}^{i, j, k}(x, y, t),
\end{aligned}
$$

where

$$
\begin{aligned}
& f_{4}^{i, j, k}(x, y, t)=\frac{\left(\alpha_{1}+\beta_{1}+i+1\right)\left(\alpha_{1}+\beta_{1}+i+2\right)}{L_{1}^{2}} \\
& \times P_{L_{1}, i-2}^{\left(\alpha_{1}+2, \beta_{1}+2\right)}(x) P_{L_{2}, j}^{\left(\alpha_{2}, \beta_{2}\right)}(y) P_{T, k}^{\left(\alpha_{3}, \beta_{3}\right)}(t), \\
& f_{5}^{i, j, k}(x, y, t)=\frac{\left(\alpha_{2}+\beta_{2}+j+1\right)\left(\alpha_{1}+\beta_{1}+j+2\right)}{L_{2}^{2}} \\
& \times P_{L_{1}, i}^{\left(\alpha_{1}, \beta_{1}\right)}(x) P_{L_{2}, j-2}^{\left(\alpha_{2}+2, \beta_{2}+2\right)}(y) P_{T, k}^{\left(\alpha_{3}, \beta_{3}\right)}(t) .
\end{aligned}
$$


Moreover, the variable order Riemann-Liouville fractional derivatives $D_{t}^{1-\gamma(x, y, t)} \frac{\partial^{2} u(x, y, t)}{\partial x^{2}}$ and $D_{t}^{1-\gamma(x, y, t)} \frac{\partial^{2} u(x, y, t)}{\partial y^{2}}$ are given by

$$
\begin{aligned}
& D_{t}^{1-\gamma(x, y, t)} \frac{\partial^{2} u(x, y, t)}{\partial x^{2}}=\sum_{i=0}^{N} \sum_{j=0}^{M} \sum_{j=0}^{K} a_{i, j, k} f_{6}^{i, j, k}(x, y, t), \\
& D_{t}^{1-\gamma(x, y, t)} \frac{\partial^{2} u(x, y, t)}{\partial y^{2}}=\sum_{i=0}^{N} \sum_{j=0}^{M} \sum_{j=0}^{K} a_{i, j, k} f_{7}^{i, j, k}(x, y, t),
\end{aligned}
$$

where

$$
\begin{aligned}
& f_{6}^{i, j, k}(x, y, t)= \frac{\left(\alpha_{1}+\beta_{1}+i+1\right)\left(\alpha_{1}+\beta_{1}+i+2\right)}{L_{1}^{2}} P_{L_{1}, i-2}^{\left(\alpha_{1}+2, \beta_{1}+2\right)}(x) \\
& \times P_{L_{2}, j}^{\left(\alpha_{2}, \beta_{2}\right)}(y) D_{t}^{1-\gamma(x, y, t)} P_{T, k}^{\left(\alpha_{3}, \beta_{3}\right)}(t) \\
& f_{7}^{i, j, k}(x, y, t)=\frac{\left(\alpha_{2}+\beta_{2}+j+1\right)\left(\alpha_{1}+\beta_{1}+j+2\right)}{L_{2}^{2}} P_{L_{1}, i}^{\left(\alpha_{1}, \beta_{1}\right)}(x) \\
& \times P_{L_{2}, j-2}^{\left(\alpha_{2}+2, \beta_{2}+2\right)}(y) D_{t}^{1-\gamma(x, y, t)} P_{T, k}^{\left(\alpha_{3}, \beta_{3}\right)}(t) .
\end{aligned}
$$

Therefore, adopting (4.2)-(4.3), enable one to write (4.1) in the form:

$$
\begin{gathered}
\sum_{i=0}^{N} \sum_{j=0}^{M} \sum_{k=0}^{K} a_{i, j, k} f_{8}^{i, j, k}(x, y, t)=H\left(\sum_{i=0}^{N} \sum_{j=0}^{M} \sum_{k=0}^{K} a_{i, j, k} f_{0}^{i, j, k}(x, y, t), x, y, t\right), \\
(x, y, t) \in\left[0, L_{1}\right] \times\left[0, L_{2}\right] \times[0, T],
\end{gathered}
$$

where

$$
f_{8}^{i, j, k}(x, y, t)=f_{3}^{i, j, k}(x, y, t)-f_{6}^{i, j, k}(x, y, t)-f_{7}^{i, j, k}(x, y, t)+f_{1}^{i, j, k}(x, y, t) .
$$

Moreover, the collocation treatments of the initial-boundary conditions immediately give

$$
\begin{aligned}
u(x, y, 0) & =\sum_{i=0}^{N} \sum_{j=0}^{M} \sum_{k=0}^{K} a_{i, j, k} f_{0}^{i, j, k}(x, y, 0)=g_{0}(x, y), \\
u(0, y, t) & =\sum_{i=0}^{N} \sum_{j=0}^{M} \sum_{k=0}^{K} a_{i, j, k} f_{0}^{i, j, k}(0, y, t)=g_{1}(y, t), \\
u\left(L_{1}, y, t\right) & =\sum_{i=0}^{N} \sum_{j=0}^{M} \sum_{k=0}^{K} a_{i, j, k} f_{0}^{i, j, k}\left(L_{1}, y, t\right)=g_{2}(y, t), \\
u(x, 0, t) & =\sum_{i=0}^{N} \sum_{j=0}^{M} \sum_{k=0}^{K} a_{i, j, k} f_{0}^{i, j, k}(x, 0, t)=g_{3}(x, t), \\
u\left(x, L_{2}, t\right) & =\sum_{i=0}^{N} \sum_{j=0}^{M} \sum_{k=0}^{K} a_{i, j, k} f_{0}^{i, j, k}\left(x, L_{2}, t\right)=g_{4}(x, t) .
\end{aligned}
$$


In the proposed method, the residual of (4.1) is set to be zero at $(N-1) \times$ $(M-1) \times K$ of collocation points

$$
\sum_{i=0}^{N} \sum_{j=0}^{M} \sum_{k=0}^{K} F_{r, s, \varsigma}^{i, j, k} a_{i, j, k}=H\left(\sum_{i=0}^{N} \sum_{j=0}^{M} \sum_{k=0}^{K} a_{i, j, k} \chi_{r, s, \varsigma}, x_{L_{1}, N, r}^{\left(\alpha_{1}, \beta_{1}\right)}, y_{L_{2}, M, s}^{\left(\alpha_{2}, \beta_{2}\right)}, t_{T, M, \varsigma}^{\left(\alpha_{3}, \beta_{3}\right)}\right)
$$

$r=1, \ldots, N-1, s=1, \ldots, M-1, \varsigma=1, \ldots, K$, where

$$
F_{r, s, \varsigma}^{i, j, k}=f_{8}^{i, j, k}\left(x_{L_{1}, N, r}^{\left(\alpha_{1}, \beta_{1}\right)}, y_{L_{2}, M, s}^{\left(\alpha_{2}, \beta_{2}\right)}, t_{T, M, \varsigma}^{\left(\alpha_{3}, \beta_{3}\right)}\right), \chi_{r, s, \varsigma}=f_{0}^{i, j, k}\left(x_{L_{1}, N, r}^{\left(\alpha_{1}, \beta_{1}\right)}, y_{L_{2}, M, s}^{\left(\alpha_{2}, \beta_{2}\right)}, t_{T, M, \varsigma}^{\left(\alpha_{3}, \beta_{3}\right)}\right),
$$

and from the initial conditions, we have, namely $(1+N+2 K N+M(1+2 K+N))$ algebraic equations

$$
\begin{aligned}
& \sum_{i=0}^{N} \sum_{j=0}^{M} \sum_{k=0}^{K} a_{i, j, k} f_{0}^{i, j, k}\left(x_{L_{1}, N, r}^{\left(\alpha_{1}, \beta_{1}\right)}, y_{L_{2}, M, s}^{\left(\alpha_{2}, \beta_{2}\right)}, 0\right)=g_{0}\left(x_{L_{1}, N, r}^{\left(\alpha_{1}, \beta_{1}\right)}, y_{L_{2}, M, s}^{\left(\alpha_{2}, \beta_{2}\right)}\right), \\
& \quad r=1, \ldots, N-1, \quad s=1, \ldots, M-1, \\
& \sum_{i=0}^{N} \sum_{j=0}^{M} \sum_{k=0}^{K} a_{i, j, k}^{i, j} f_{0}^{i, j, k}\left(0, y_{L_{2}, M, s}^{\left(\alpha_{2}, \beta_{2}\right)}, t_{T, M, \varsigma}^{\left(\alpha_{3}, \beta_{3}\right)}\right)=g_{1}\left(y_{L_{2}, M, s}^{\left(\alpha_{2}, \beta_{2}\right)}, t_{T, M, \varsigma}^{\left(\alpha_{3}, \beta_{3}\right)}\right), \\
& \quad s=0, \ldots, M, \quad \varsigma=0, \ldots, K, \\
& \sum_{i=0}^{N} \sum_{j=0}^{M} \sum_{k=0}^{K} a_{i, j, k}^{i, j} f_{0}^{i, j, k}\left(L_{1}, y_{L_{2}, M, s}^{\left(\alpha_{2}, \beta_{2}\right)}, t_{T, M, \varsigma}^{\left(\alpha_{3}, \beta_{3}\right)}\right)=g_{2}\left(y_{L_{2}, M, s}^{\left(\alpha_{2}, \beta_{2}\right)}, t_{T, M, \varsigma}^{\left(\alpha_{3}, \beta_{3}\right)}\right), \\
& \quad s=0, \ldots, M, \quad \varsigma=0, \ldots, K, \\
& \sum_{i=0}^{N} \sum_{j=0}^{M} \sum_{k=0}^{K} a_{i, j, k}^{i, j} f_{0}^{i, j, k}\left(x_{L_{1}, N, r}^{\left(\alpha_{1}, \beta_{1}\right)}, 0, t_{T, M, \varsigma}^{\left(\alpha_{3}, \beta_{3}\right)}\right)=g_{3}\left(x_{L_{1}, N, r}^{\left(\alpha_{1}, \beta_{1}\right)}, t_{T, M, \varsigma}^{\left(\alpha_{3}, \beta_{3}\right)}\right), \\
& \quad r=1, \ldots, N-1, \quad \varsigma=0, \ldots, K, \\
& \sum_{i=0}^{N} \sum_{j=0}^{M} \sum_{k=0}^{K} a_{i, j, k}^{i, j} f_{0}^{i, j, k}\left(x_{L_{1}, N, r}^{\left(\alpha_{1}, \beta_{1}\right)}, L_{2}, t_{T, M, \varsigma}^{\left(\alpha_{3}, \beta_{3}\right)}\right)=g_{4}\left(x_{L_{1}, N, r}^{\left(\alpha_{1}, \beta_{1}\right)}, t_{T, M, \varsigma}^{\left(\alpha_{3}, \beta_{3}\right)}\right), \\
& r=1, \ldots, N-1, \quad \varsigma=0, \ldots, K,
\end{aligned}
$$

and this in turn, yields $(M+1) \times(N+1) \times(K+1)$ nonlinear algebraic equations

$$
\begin{aligned}
& \sum_{i=0}^{N} \sum_{j=0}^{M} \sum_{k=0}^{K} F_{r, s, \varsigma}^{i, j, k} a_{i, j, k}=H\left(\sum_{i=0}^{N} \sum_{j=0}^{M} \sum_{k=0}^{K} a_{i, j, k} \chi_{r, s, \varsigma}, x_{L_{1}, N, r}^{\left(\alpha_{1}, \beta_{1}\right)}, y_{L_{2}, M, s}^{\left(\alpha_{2}, \beta_{2}\right)}, t_{T, M, \varsigma}^{\left(\alpha_{3}, \beta_{3}\right)}\right), \\
& \quad r=1, \ldots, N-1, \quad s=1, \ldots, M-1, \varsigma=1, \ldots, K, \\
& \sum_{i=0}^{N} \sum_{j=0}^{M} \sum_{k=0}^{K} a_{i, j, k} f_{0}^{i, j, k}\left(x_{L_{1}, N, r}^{\left(\alpha_{1}, \beta_{1}\right)}, y_{L_{2}, M, s}^{\left(\alpha_{2}, \beta_{2}\right)}, 0\right)=g_{0}\left(x_{L_{1}, N, r}^{\left(\alpha_{1}, \beta_{1}\right)}, y_{L_{2}, M, s}^{\left(\alpha_{2}, \beta_{2}\right)}\right), \\
& \quad r=1, \ldots, N-1, \quad s=1, \ldots, M-1,
\end{aligned}
$$




$$
\begin{aligned}
& \sum_{i=0}^{N} \sum_{j=0}^{M} \sum_{k=0}^{K} a_{i, j, k}^{i, j} f_{0}^{i, j, k}\left(0, y_{L_{2}, M, s}^{\left(\alpha_{2}, \beta_{2}\right)}, t_{T, M, \varsigma}^{\left(\alpha_{3}, \beta_{3}\right)}\right)=g_{1}\left(y_{L_{2}, M, s}^{\left(\alpha_{2}, \beta_{2}\right)}, t_{T, M, \varsigma}^{\left(\alpha_{3}, \beta_{3}\right)}\right), \\
& s=0, \ldots, M, \quad \varsigma=0, \ldots, K, \\
& \sum_{i=0}^{N} \sum_{j=0}^{M} \sum_{k=0}^{K} a_{i, j, k}^{i, j} f_{0}^{i, j, k}\left(L_{1}, y_{L_{2}, M, s}^{\left(\alpha_{2}, \beta_{2}\right)}, t_{T, M, \varsigma}^{\left(\alpha_{3}, \beta_{3}\right)}\right)=g_{2}\left(y_{L_{2}, M, s}^{\left(\alpha_{2}, \beta_{2}\right)}, t_{T, M, \varsigma}^{\left(\alpha_{3}, \beta_{3}\right)}\right), \\
& s=0, \ldots, M, \quad \varsigma=0, \ldots, K, \\
& \sum_{i=0}^{N} \sum_{j=0}^{M} \sum_{k=0}^{K} a_{i, j, k}^{i, j} f_{0}^{i, j, k}\left(x_{L_{1}, N, r}^{\left(\alpha_{1}, \beta_{1}\right)}, 0, t_{T, M, \varsigma}^{\left(\alpha_{3}, \beta_{3}\right)}\right)=g_{3}\left(x_{L_{1}, N, r}^{\left(\alpha_{1}, \beta_{1}\right)}, t_{T, M, \varsigma}^{\left(\alpha_{3}, \beta_{3}\right)}\right), \\
& r=1, \ldots, N-1, \quad \varsigma=0, \ldots, K, \\
& \sum_{i=0}^{N} \sum_{j=0}^{M} \sum_{k=0}^{K} a_{i, j, k}^{i, j} f_{0}^{i, j, k}\left(x_{L_{1}, N, r}^{\left(\alpha_{1}, \beta_{1}\right)}, L_{2}, t_{T, M, \varsigma}^{\left(\alpha_{3}, \beta_{3}\right)}\right)=g_{4}\left(x_{L_{1}, N, r}^{\left(\alpha_{1}, \beta_{1}\right)}, t_{T, M, \varsigma}^{\left(\alpha_{3}, \beta_{3}\right)}\right), \\
& r=1, \ldots, N-1, \quad \varsigma=0, \ldots, K .
\end{aligned}
$$

The previous system of nonlinear algebraic equations can be easily solved. After the coefficients $a_{i, j, k}$ are determined, we compute the approximate solution $u_{N, M, K}(x, y, t)$ at any value of $(x, y, t)$ in the given domain.

\section{$5 \quad$ Numerical results and comparisons}

This section listed several numerical examples to demonstrate the accuracy of the proposed method. Also, we compare our numerical results with the existing numerical results [7]. The obtained results of these examples show that the proposed method, by selecting a few number nodes, has high level of accuracy.

The difference between the measured value of approximate solution and exact solution is defined absolute error (AE), given by

$$
E(x, t)=\left|u(x, t)-u_{N, M}(x, t)\right|
$$

where $u(x, t)$ and $u_{N, M}(x, t)$ are the exact and the approximate solutions at the point $(x, t)$, respectively.

Moreover, the maximum absolute error (MAE) is given by

$$
M_{E}=\max \{E(x, t): \forall(x, t) \in[0, L] \times[0, T]\} .
$$




\subsection{One-dimensional space of VO-NGIADE}

Example 1. We start with the VO-NGIADE in the following form [7]:

$$
\begin{aligned}
& \frac{\partial u(x, t)}{\partial t}+\frac{\partial u(x, t)}{\partial x}=D_{t}^{1-\gamma(x, t)}\left(\frac{\partial^{2} u(x, t)}{\partial x^{2}}\right)+u(x, t)-(u(x, t))^{2} \\
& \quad+t e^{x}\left(2+t^{3} e^{x}-\frac{2 t^{\gamma(x, t)}}{\Gamma(\gamma(x, t)+2)}\right), \\
& u(0, t)=t^{2}, \quad u(1, t)=e t^{2}, \quad t \in[0,1], \\
& u(x, 0)=0, \quad x \in[0,1],
\end{aligned}
$$

where $(x, t) \in[0,1] \times[0,1]$, and $\gamma(x, t)=\frac{1}{300}(10-t x)$, knowing that the exact solution is given by $u(x, t)=t^{2} e^{x}$.

In Table 1, we display a comparison based on the MAEs between our results (with various choices of $N, M, \alpha_{1}, \beta_{1}, \alpha_{2}$, and $\beta_{2}$ ) and the finite difference method (FDM) [7].

Table 1. The MAEs of Example 1.

\begin{tabular}{lllll}
\hline & \multicolumn{4}{c}{ Our method with several choices of $N, M$} \\
\cline { 2 - 5 }$\left(\alpha_{1}, \beta_{1}, \alpha_{2}, \beta_{2}\right)$ & 2 & 6 & 10 & 14 \\
\hline$(0,0,0,0)$ & $1.36284 \times 10^{-3}$ & $5.42912 \times 10^{-8}$ & $2.66454 \times 10^{-14}$ & $6.66134 \times 10^{-16}$ \\
$\left(\frac{1}{2}, \frac{1}{2}, 0,0\right)$ & $1.36284 \times 10^{-3}$ & $4.08291 \times 10^{-8}$ & $2.62013 \times 10^{-14}$ & $4.44089 \times 10^{-16}$ \\
$\left(-\frac{1}{2}, 0, \frac{1}{2}, \frac{1}{2}\right)$ & $1.26147 \times 10^{-2}$ & $7.07546 \times 10^{-8}$ & $3.59712 \times 10^{-14}$ & $6.66134 \times 10^{-16}$ \\
\hline
\end{tabular}

FDM [7] with several choices of $h_{t}, h_{x}$

\begin{tabular}{llll}
\hline & $h_{t}=h_{x}^{2}=\frac{1}{16}$ & $h_{t}=h_{x}^{2}=\frac{1}{64}$ & $h_{t}=h_{x}^{2}=\frac{1}{256}$ \\
\hline- & $5.5308 \times 10^{-4}$ & $1.4567 \times 10^{-4}$ & $6.1896 \times 10^{-5}$ \\
\hline
\end{tabular}

Figure 1 display the space-time graph of the AEs with $N=M=14$, and $\alpha_{1}=\beta_{1}=\alpha_{2},=\beta_{2}=0$. While, Figure 2 compare graphically the curves of numerical and exact solutions of Example 1 for the different values of $x$ at $N=M=14, \alpha_{1}=\beta_{1}=\frac{1}{2}$ and $\alpha_{2},=\beta_{2}=0$.

The $t$-direction curve of AEs of Example 1 for $N=M=14$, and $\alpha_{1}=$ $\beta_{1}=\alpha_{2}=\beta_{2}=0$, is displayed in Figure 3. Moreover, we plot in Figure 4 the logarithmic graphs of MAEs (i.e., $\log _{10} M_{E}$ ) obtained by the present method with different values of $(N=M=2,4,6, \cdots, 14)$ at three choices of $\alpha_{1}, \beta_{1}, \alpha_{2}$, and $\beta_{2}$. This demonstrates that the proposed method leads to an accurate approximation and yields exponential convergence rates.

Example 2. Consider the VO-NGIADE in the following form [7]:

$$
\begin{aligned}
& \frac{\partial u(x, t)}{\partial t}+\frac{\partial u(x, t)}{\partial x}=D_{t}^{1-\gamma(x, t)}\left(\frac{\partial^{2} u(x, t)}{\partial x^{2}}\right)+u(x, t)-(u(x, t))^{2} \\
& \quad+t e^{x}\left(2+t^{3} e^{x}-\frac{2 t^{\gamma(x, t)}}{\Gamma(\gamma(x, t)+2)}\right), \\
& \begin{array}{ll}
u(0, t)=t^{2}, & u(1, t)=e t^{2}, \quad t \in[0,1], \\
u(x, 0)=0, & x \in[0,1],
\end{array}
\end{aligned}
$$




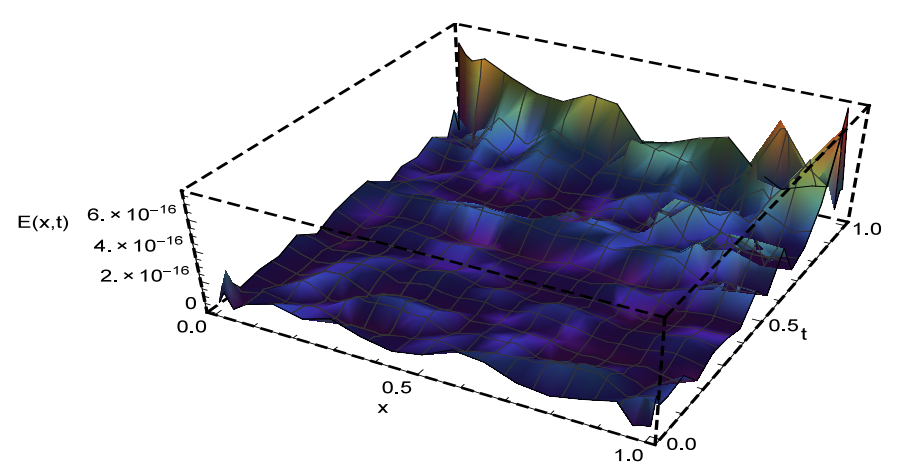

Figure 1. Space-time graph of the AEs of Example 1.

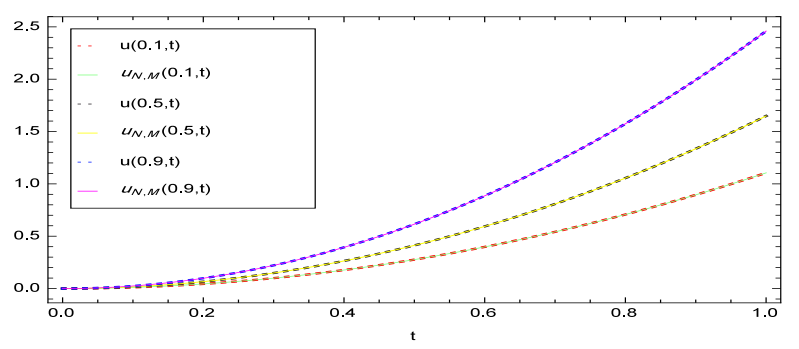

Figure 2. $t$-direction curves of exact and numerical solutions of Example 1.

where $(x, t) \in[0,1] \times[0,1]$, and

$$
\gamma(x, t)=\frac{1}{500}\left((t x)^{2}-\sin ^{3}(t x)+\cos ^{4}(t x)+16\right)
$$

is the exact solution is given by $u(x, t)=t^{2} e^{x}$.

Table 2, displays the MAEs using the present method together with the results obtained in [7] for different choices of $N, M, \alpha_{1}, \beta_{1}, \alpha_{2}$, and $\beta_{2}$. From the results of this example, we observe that the approximate solution obtained by our method is more better than those obtained in [7].

Example 3. Here, we test the VO-NGIADE in the following form:

$$
\begin{aligned}
& \frac{\partial u(x, t)}{\partial t}+\frac{\partial u(x, t)}{\partial x}=D_{t}^{1-\gamma(x, t)}\left(\frac{\partial^{2} u(x, t)}{\partial x^{2}}\right)+u(x, t)-(u(x, t))^{2}+f(x, t), \\
& u(0, t)=t^{2.8}, \quad u(1, t)=e t^{2.8}, \quad t \in[0,1], \\
& u(x, 0)=0, \quad x \in[0,1],
\end{aligned}
$$

where $(x, t) \in[0,1] \times[0,1]$, and $f(x, t)$ is a given function such that $\gamma(x, t)=$ $\frac{1}{600}\left(20-e^{t x}\right)$, and the exact solution is $u(x, t)=t^{2.8} e^{x}$.

The MAEs for Example 3 are listed in Table 3 at different choice of $N, M$, $\alpha_{1}, \beta_{1}, \alpha_{2}$, and $\beta_{2}$. This table confirm the high accuracy of the present scheme. 


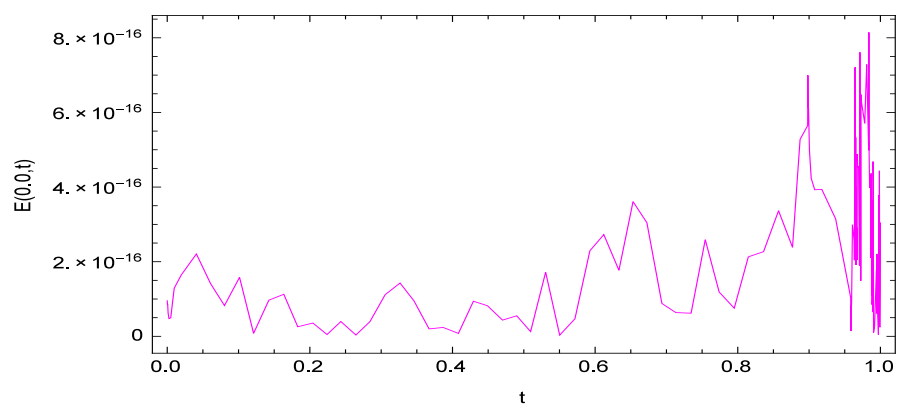

Figure 3. $t$-direction curve of the AEs of Example 1.

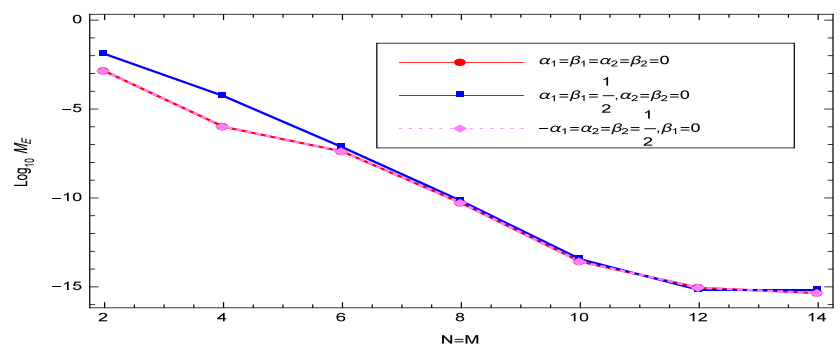

Figure 4. $M_{E}$ convergence of Example 1.

Example 4. Here, we test the VO-NGIADE in the following form:

$$
\begin{aligned}
& \frac{\partial u(x, t)}{\partial t}+\frac{\partial u(x, t)}{\partial x}=D_{t}^{1-\gamma(x, t)}\left(\frac{\partial^{2} u(x, t)}{\partial x^{2}}\right)+u(x, t)-(u(x, t))^{2} \\
& +t e^{-2 x}\left(-\frac{2 e^{x} t^{\gamma(x, t)}}{\Gamma(\gamma(x, t)+2)}+t^{3}-2(t-1) e^{x}\right) \\
& u(0, t)=t^{2}, \quad u(1, t)=e t^{2}, \quad t \in[0,10] \\
& u(x, 0)=0, \quad x \in[0,1]
\end{aligned}
$$

where $(x, t) \in[0,1] \times[0,10], \gamma(x, t)=\frac{1}{500}\left((t x)^{2}-\sin ^{3}(t x)+\cos ^{4}(t x)+266\right)$, knowing that the exact solution is given by $u(x, t)=t^{2} e^{-x}$.

The MAEs for Example 4 are listed in Table 4 at different choice of $N, M$, $\alpha_{1}, \beta_{1}, \alpha_{2}$, and $\beta_{2}$.

The space-time graph of AEs of Example 4, for $N=10, M=20$, and $\alpha_{1}=\beta_{1}=\alpha_{2},=\beta_{2}=0$, is sketched in Figure 5 . 
Table 2. The MAEs of Example 2.

\begin{tabular}{lllll}
\hline & \multicolumn{4}{c}{ Our method with several choices of $N, M$} \\
\cline { 2 - 5 }$\left(\alpha_{1}, \beta_{1}, \alpha_{2}, \beta_{2}\right)$ & 4 & 8 & 10 \\
\hline$(0,0,0,0)$ & $3.0160 \times 10^{-5}$ & $5.4289 \times 10^{-8}$ & $4.6928 \times 10^{-11}$ & $2.5757 \times 10^{-14}$ \\
$\left(0, \frac{1}{2}, 1, \frac{1}{2}\right)$ & $3.5922 \times 10^{-5}$ & $5.7451 \times 10^{-8}$ & $7.9964 \times 10^{-11}$ & $4.4631 \times 10^{-14}$ \\
$\left(1, \frac{1}{2},-\frac{1}{2}, 0\right)$ & $4.8971 \times 10^{-5}$ & $7.9121 \times 10^{-8}$ & $1.1243 \times 10^{-10}$ & $7.7716 \times 10^{-14}$ \\
\hline
\end{tabular}

FDM [7] with several choices of $h_{t}, h_{x}$

\begin{tabular}{llll}
\hline- & - & $h_{t}=h_{x}^{2}=\frac{1}{16}$ & $h_{t}=h_{x}^{2}=\frac{1}{64}$ \\
\hline- & - & $5.6574 \times 10^{-4}$ & $1.4910 \times 10^{-4}$ \\
\hline
\end{tabular}

Table 3. The MAEs of Example 3.

\begin{tabular}{llllll}
\hline & \multicolumn{5}{c}{ Our method with several choices of $(N, M)$} \\
\cline { 2 - 6 }$\left(\alpha_{1}, \beta_{1}, \alpha_{2}, \beta_{2}\right)$ & $(8,4)$ & $(8,8)$ & $(8,16)$ & $(8,20)$ & $(8,24)$ \\
\hline$(0,0,0,0)$ & $9.92325 e-4$ & $1.4186 e-5$ & $1.9261 e-7$ & $4.7128 e-8$ & $1.4803 e-8$ \\
$\left(\frac{1}{2}, \frac{1}{2}, \frac{1}{2}, \frac{1}{2}\right)$ & $1.46369 e-3$ & $2.4485 e-5$ & $3.7493 e-7$ & $9.4460 e-8$ & $3.0290 e-8$ \\
$\left(-\frac{1}{2},-\frac{1}{2}, \frac{1}{2}, \frac{1}{2}\right)$ & $1.46369 e-3$ & $2.4485 e-5$ & $3.7493 e-7$ & $9.4460 e-8$ & $3.0290 e-8$ \\
\hline
\end{tabular}

\subsection{Two-dimensional space of VO-NGIADE}

Example 5. We consider the following two-dimensional space VO-NGIADE

$$
\begin{aligned}
& \frac{\partial u(x, y, t)}{\partial t}+\frac{\partial u(x, y, t)}{\partial x}=D_{t}^{1-\gamma(x, t)}\left(\frac{\partial^{2} u(x, y, t)}{\partial x^{2}}+\frac{\partial^{2} u(x, y, t)}{\partial y^{2}}\right) \\
& +u(x, y, t)-(u(x, y, t))^{2}+f(x, t), \\
& u(0, y, t)=t^{2} \sin (y), \quad u(1, y, t)=t^{2} \sin (1+y), \quad(y, t) \in[0,1] \times[0,1], \\
& u(x, 0, t)=t^{2} \sin (x), \quad u(x, 1, t)=t^{2} \sin (1+x), \quad(x, t) \in[0,1] \times[0,1], \\
& u(x, y, 0)=0, \quad(x, y) \in[0,1] \times[0,1],
\end{aligned}
$$

$(x, y, t) \in[0,1] \times[0,1] \times[0,1], \gamma(x, y, t)=\frac{1}{400}(t(-x) y+\cos (t x y)+13)$ and $f(x, t)=t\left(\frac{4 \sin (x+y) t^{\gamma(x, y, t)}}{\Gamma(\gamma(x, y, t)+2)}+\sin (x+y)\left(t^{3} \sin (x+y)-t+2\right)+2 t \cos (x+y)\right)$, knowing that the exact solution is given by $u(x, y, t)=t^{2} \sin (x+y)$.

The MAEs obtained by our method are summarized in Table 5 , for several choices of $N, M, K, \alpha_{1}, \beta_{1}, \alpha_{2}, \beta_{2}, \alpha_{3}$, and $\beta_{3}$. This results confirm the high accuracy of the present scheme.

The three-dimensional graph of the AEs of Example 5 at $t=\frac{1}{2}, N=$ $M=8, K=4, \alpha_{1}=\frac{1}{2}, \beta_{1}=0, \alpha_{2}=\frac{1}{2}, \beta_{2}=0$, and $\alpha_{3}=\beta_{3}=\frac{1}{2}$ is displayed in Figure 6. In addition, the curve of the AEs of Example 5, at $y=t=\frac{1}{2}$, is displayed in Figure 7 with the choice $N=M=8, K=4$ and $\alpha_{1}=\frac{1}{2}, \beta_{1}=0, \alpha_{2}=\frac{1}{2}, \beta_{2}=0, \alpha_{3}=\beta_{3}=\frac{1}{2}$. 
Table 4. The MAEs of Example 4.

\begin{tabular}{llllll}
\hline & \multicolumn{5}{c}{ Our method with several choices of $\left(\alpha_{1}, \beta_{1}, \alpha_{2}, \beta_{2}\right)$} \\
\cline { 2 - 6 }$(N, M)$ & $\left(\frac{1}{2}, \frac{1}{2}, \frac{1}{2}, \frac{1}{2}\right)$ & $\left(-\frac{1}{2},-\frac{1}{2}, \frac{1}{2}, \frac{1}{2}\right)$ & $\left(\frac{1}{2}, \frac{1}{2}, 0,0\right)$ & $\left(-\frac{1}{2},-\frac{1}{2}, 1,0\right)$ & $(0,0,0,0)$ \\
\hline$(5,5)$ & $3.6056 e-5$ & $7.9412 \times 10^{-5}$ & $3.6056 e-5$ & $7.9405 \times 10^{-5}$ & $5.5766 \times 10^{-5}$ \\
$(10,20)$ & $7.3896 e-13$ & $1.4495 \times 10^{-12}$ & $7.9581 e-13$ & $1.4353 \times 10^{-12}$ & $1.0090 \times 10^{-12}$ \\
\hline
\end{tabular}

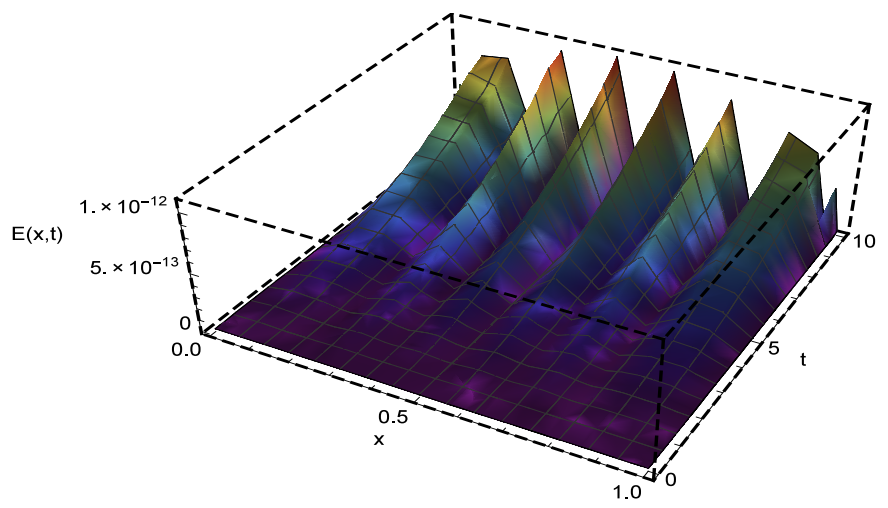

Figure 5. Space-time graph of the AEs of Example 4.

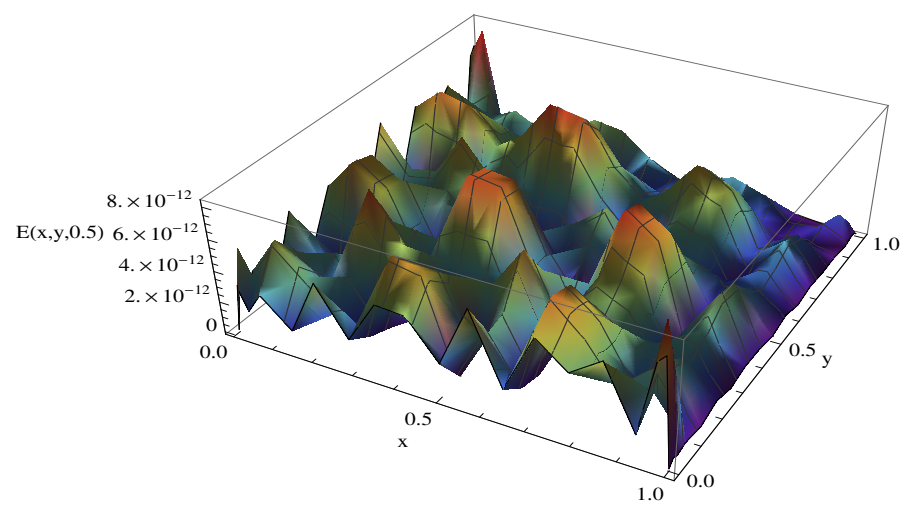

Figure 6. Space graph of the AEs for Example 5.

Example 6. Finally, we introduce the following two-dimensional VO-NGIADE

$$
\begin{aligned}
& \frac{\partial u(x, y, t)}{\partial t}+\frac{\partial u(x, y, t)}{\partial x}=D_{t}^{1-\gamma(x, t)}\left(\frac{\partial^{2} u(x, y, t)}{\partial x^{2}}+\frac{\partial^{2} u(x, y, t)}{\partial y^{2}}\right) \\
& +u(x, y, t)-(u(x, y, t))^{2}+f(x, t), \\
& u(0, y, t)=t^{2} e^{y}, \quad u(1, y, t)=t^{2} e^{y+1}, \quad(y, t) \in[0,1] \times[0,1], \\
& u(x, 0, t)=t^{2} e^{x}, u(x, 1, t)=t^{2} e^{x+1}, \quad(x, t) \in[0,1] \times[0,1], \\
& u(x, y, 0)=0, \quad(x, y) \in[0,1] \times[0,1],
\end{aligned}
$$


Table 5. The MAEs of Example 5.

\begin{tabular}{lllll}
\hline \multirow{2}{*}{$\left(\alpha_{1}, \beta_{1}, \alpha_{2}, \beta_{2}, \alpha_{3}, \beta_{3}\right)$} & \multicolumn{4}{c}{ Our method with several choices of $(N, M, K)$} \\
\cline { 2 - 5 } & $(2,2,2)$ & $(4,4,4)$ & $(6,6,6)$ & $(8,8,4)$ \\
\hline$(0,0,0,0,0,0)$ & $2.8713 e-3$ & $2.50895 \times 10^{-5}$ & $5.23130 \times 10^{-8}$ & $4.2599 e-11$ \\
$\left(\frac{1}{2}, 0, \frac{1}{2}, 0, \frac{1}{2}, \frac{1}{2}\right)$ & $2.3442 e-3$ & $2.49389 \times 10^{-5}$ & $3.82665 \times 10^{-8}$ & $3.4212 e-11$ \\
$\left(\frac{1}{2},-\frac{1}{2}, 0,0, \frac{1}{2}, \frac{1}{2}\right)$ & $1.0031 e-2$ & $3.636267 \times 10^{-5}$ & $4.15992 \times 10^{-8}$ & $4.4601 e-11$ \\
\hline
\end{tabular}

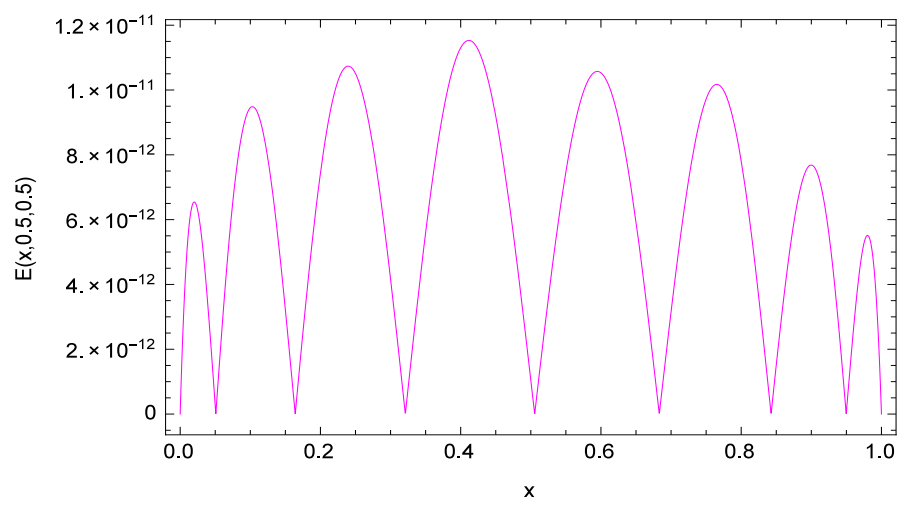

Figure 7. The AEs versus $x$ of Example 5 .

where $(x, y, t) \in[0,1] \times[0,1] \times[0,1], \gamma(x, y, t)=\frac{1}{300}(150-t x y)$ and $f(x, t)=$ $t e^{x+y}\left(t+2-\frac{4 t^{\gamma(x, y, t)}}{\Gamma(\gamma(x, y, t)+2)}+t^{3} e^{x+y}\right)$, knowing that the exact solution is given by $u(x, y, t)=t^{2} e^{x+y}$.

Table 6 lists the MAEs obtained by using the proposed method for various choices of $N, M, K, \alpha_{1}, \beta_{1}, \alpha_{2}, \beta_{2}, \alpha_{3}$, and $\beta_{3}$. The numerical results presented in this table show that the results are vary accurate for small value of $N, M$ and $K$.

Table 6. The MAEs of Example 6.

\begin{tabular}{lllll}
\hline \multirow{2}{*}{$\left(\alpha_{1}, \beta_{1}, \alpha_{2}, \beta_{2}, \alpha_{3}, \beta_{3}\right)$} & \multicolumn{4}{c}{ Our method with several choices of $(N, M, K)$} \\
\cline { 2 - 5 } & $(2,2,2)$ & $(4,4,4)$ & $(6,6,6)$ & $(8,8,4)$ \\
\hline$(0,0,0,0,0,0)$ & $1.0934 \times 10^{-3}$ & $1.3013 \times 10^{-4}$ & $2.4714 \times 10^{-7}$ & $1.9615 e-10$ \\
$\left(\frac{1}{2}, 0, \frac{1}{2}, 0, \frac{1}{2}, \frac{1}{2}\right)$ & $2.1502 \times 10^{-2}$ & $7.1109 \times 10^{-5}$ & $1.5411 \times 10^{-7}$ & $2.6118 e-10$ \\
$\left(\frac{1}{2},-\frac{1}{2}, 0,0, \frac{1}{2}, \frac{1}{2}\right)$ & $4.7764 \times 10^{-2}$ & $9.9759 \times 10^{-5}$ & $1.9505 \times 10^{-7}$ & $3.3079 e-10$ \\
\hline
\end{tabular}

\section{Conclusions}

By means of SJ-GL-C and SJ-GR-C schemes, we have introduced a spacetime spectral algorithm for solving VO-NGIADs . According to the numerical results obtained above, we can concluded the high accuracy of our technique. 
Comparisons between our approximate solutions of the problems, with their exact solutions or with the approximate solutions achieved by other methods, were also included to confirm the validity and accuracy of the new scheme.

\section{References}

[1] D. Baleanu and K. Sayevand. Performance evaluation of matched asymptotic expansions for fractional differential equations with multi-order. B. Math. Soc. Sci. Math., 59(1):3-12, 2016.

[2] A.H. Bhrawy. An efficient Jacobi pseudospectral approximation for nonlinear complex generalized Zakharov system. Appl. Math. Comput., 247:30-46, 2014. https://doi.org/10.1016/j.amc.2014.08.062.

[3] A.H. Bhrawy and M.A. Zaky. Numerical simulation for two-dimensional variableorder fractional nonlinear cable equation. Nonlinear Dyn., 80(1):101-116, 2015. https://doi.org/10.1007/s11071-014-1854-7.

[4] A.H. Bhrawy, M.A. Zaky and J.A. Tenreiro Machado. Efficient Legendre spectral tau algorithm for solving the two-sided space-time Caputo fractional advection-dispersion equation. J. Vib. Control., 22(8):2053-2068, 2015. https://doi.org/10.1177/1077546314566835.

[5] C-M. Chen, F. Liu, V. Anh and I. Turner. Numerical methods for solving a twodimensional variable-order anomalous subdiffusion equation. Math. Math. Comput., 81(277):345-366, 2012. https://doi.org/10.1090/S0025-5718-2011-024476.

[6] C.M. Chen. Numerical methods for solving a two-dimensional variableorder modified diffusion equation. Appl. Math. Comput., 225:62-78, 2013. https://doi.org/10.1016/j.amc.2013.08.064.

[7] C.M. Chen, F. Liu, V. Anh and I. Turner. Numerical simulation for the variable-order Galilei invariant advection diffusion equation with a nonlinear source term. Appl. Math. Comput., 217(12):5729-5742, 2011. https://doi.org/10.1016/j.amc.2010.12.049.

[8] C.M. Chen, F. Liu, I. Turner and V. Anh. Numerical methods with fourthorder spatial accuracy for variable-order nonlinear Stokes first problem for a heated generalized second grade fluid. Comput. Math. Appl., 62(3):971-986, 2011. https://doi.org/10.1016/j.camwa.2011.03.065.

[9] C.F.M. Coimbra. Mechanics with variable-order differential operators. Ann. Phys., 12(11-12):692-703, 2003. https://doi.org/10.1002/andp.200310032.

[10] E. H Doha, A H Bhrawy and M A. Abdelkawy. An accurate Jacobi pseudo-spectral algorithm for parabolic partial differential equations with nonlocal boundary conditions. J. Comput. Nonlinear Dyn., 10(2):021016, 2015. https://doi.org/10.1115/1.4026930.

[11] V.J. Ervin and J.P.Roop. Variational solution of fractional advection-dispersion equations on bounded domains in $\mathrm{R}^{d}$. Numer. Methods Partial Differ. Equ., 23(2):256-281, 2007. https://doi.org/10.1002/num.20169.

[12] M. Giona and H.E. Roman. Fractional diffusion equation for transport phenomena in random media. Phys. A, 185(1-4):87-97, 1992. https://doi.org/10.1016/0378-4371(92)90441-R. 
[13] H. Sun H. Sheng, C. Coopmans, Y.Q. Chen and G.W. Bohannan. Physical experimental study of variable-order fractional integrator and differentiator. In Fractional Differentiation and its Applications, 2010.

[14] R. Hilfer. Applications of Fractional Calculus in Physics. Word Scientific, Singapore, 2000. https://doi.org/10.1142/3779.

[15] J.W. Kirchner, X. Feng and C. Neal. Fractal stream chemistry and its implications for containant transport in catchments. Nature, 403:524-526, 2000. https://doi.org/10.1038/35000537.

[16] R. Lin, F. Liu, V. Anh and I. Turner. Stability and convergence of a new explicit finite-difference approximation for the variable-order nonlinear fractional diffusion equation. Appl. Math. Comput., 212(2):435-445, 2009. https://doi.org/10.1016/j.amc.2009.02.047.

[17] C.F. Lorenzo. Initialization, conceptualization, and application in the generalized (fractional) calculus CRC Crit. Rev. Biomed. Eng., 35(6):447-553, 2007. https://doi.org/10.1615/CritRevBiomedEng.v35.i6.10.

[18] C.F. Lorenzo and T.T. Hartley. Variable order and distributed order fractional operators. Nonlinear Dyn., $\mathbf{2 9}(1): 57-98,2002$. https://doi.org/10.1023/A:1016586905654.

[19] R.L. Magin. Fractional Calculus in Bioengineering. Begell House Publishers, 2006.

[20] I. Podlubny. Fractional Differential Equations, in: Mathematics in Science and Engineering. Academic Press Inc., San Diego, CA, 1999.

[21] A. Quarteroni, C. Canuto, M.Y. Hussaini and T.A. Zang. Spectral Methods: Fundamentals in Single Domains. Springer-Verlag, New York, 2006.

[22] S.G. Samko and B. Ross. Integration and differentiation to a variable fractional order. Integral Transforms Spec. Funct., 1(4):277-300, 1993. https://doi.org/10.1080/10652469308819027.

[23] S. Shen, F. Liu and V. Anh. Numerical approximations and solution techniques for the space-time Riesz-Caputo fractional advection-diffusion equation. Numer. Algor., 56(3):383-403, 2011. https://doi.org/10.1007/s11075-010-9393-x.

[24] S. Shen, F. Liu, V. Anh, I. Turner and J. Chen. A characteristic difference method for the variable-order fractional advection-diffusion equation. J. Appl. Math. Comput., 42(1):371-386, 2013. https://doi.org/10.1007/s12190-012-06420 .

[25] H. Sheng, H. Sun, Y-Q. Chen and T. Qiu. Synthesis of multifractional Gaussian noises based on variable-order fractional operators. Signal Processing, 91(7):1645-1650, 2011. https://doi.org/10.1016/j.sigpro.2011.01.010.

[26] H. Sun, W. Chen and Y-Q. Chen. Variable-order fractional differential operators in anomalous diffusion modeling. Phys. A, 388(21):4586-4592, 2009. https://doi.org/10.1016/j.physa.2009.07.024.

[27] H.G. Sun, W. Chen, C. Li and Y-Q. Chen. Finite difference schemes for variableorder time fractional diffusion equation. Int. J. Bifurcat. Chaos., 22(4), 2012. https://doi.org/10.1142/S021812741250085X.

[28] H.G. Suna, Yong Zhangb and D.M. Reevesd W. Chenc. Use of a variable-index fractional-derivative model to capture transient dispersion in heterogeneous media. J. Contam. Hydrol., 157:47-58, 2014. https://doi.org/10.1016/j.jconhyd.2013.11.002. 
[29] C.C. Tseng. Design of variable and adaptive fractional order FIR differentiators. Signal Processing, 86(10):2554-2566, 2006. https://doi.org/10.1016/j.sigpro.2006.02.004.

[30] Y. Xu and V.S. Ertürk. A finite difference technique for solving variable-order fractional integro-differential equations. Bull. Iranian Math. Soc., 40:699-712, 2014.

[31] H. Zhang, F. Liu, M.S. Phanikumar and M.M. Meerschaert. A novel numerical method for the time variable fractional order mobile-immobile advection-dispersion model. Comput. Math. Appl., 66(5):693-701, 2013. https://doi.org/10.1016/j.camwa.2013.01.031.

[32] H. Zhang, F. Liu, P. Zhuang, I. Turner and V. Anh. Numerical analysis of a new space-time variable fractional order advection-dispersion equation. Appl. Math. Comput., 242:541-550, 2014. https://doi.org/10.1016/j.amc.2014.06.003.

[33] X. Zhao, Z.Z. Sun and G.E. Karniadakis. Second-order approximations for variable order fractional derivatives: Algorithms and applications. J. Comput. Phys., 293:184-200, 2015. https://doi.org/10.1016/j.jcp.2014.08.015.

[34] P. Zhuang, F. Liu, V. Anh and I. Turner. Numerical methods for the variableorder fractional advection-diffusion equation with a nonlinear source term. SIAM J. Numer. Anal., 47(3):1760-1781, 2009. https://doi.org/10.1137/080730597. 